\title{
Genomic organization of serratiochelin cluster in the environmental and clinical strains of Serratia marcescens
}

\author{
I. Khilyas ${ }^{1 *}$, M. Sharipova ${ }^{1}$, L. Bogomolnaya ${ }^{1,2}$ \\ ${ }^{1}$ Institute of Fundamental Medicine and Biology KFU, Kazan, Russia \\ ${ }^{2}$ Texas A\&M University Health Science Center, Bryan, Texas, USA \\ *e-mail: irina.khilyas@gmail.com
}

Key words: siderophores, serratiochelin, Serratia

Motivation and Aim: Bacterial siderophores are small secondary metabolites with a strong ferric iron-binding capacity. Availability of iron in the mammal hosts during infection is extremely limited for support of bacterial survival. The non-ribosomal peptide synthetase (NRPS) - mediated combinatorial biosynthesis of siderophores is induced by the iron starvation and functions to replenish iron supply in the bacterial cells [1]. Thus, siderophores play an essential role as a virulence factor of pathogenic bacteria. A goal of this study was to identify the serratiochelin gene cluster in the genomes of the environmental and clinical strains of S. marcescens, SM6 and SR 41-8000.

Methods and Algorithms: S. marcescens genomes were analyzed for the presence of siderophores-encoding gene clusters using AntiSmash software and using RAST platform for gene annotation [2].

Results: Genome analysis of S. marcescens strains Sm6 and SR41-8000 showed the presence of NRPS modules SM6_200-203 and SR41_3114-3117, respectively. Closely related bacteria Serratia sp. V4 was recently showed to synthetize siderophores serratiochelines A, B and C [3]. The BLAST analysis of amino acid sequences of identified NRPS S. marcescens SM6 and SR 41-8000 against serratiochelin NRPS Serratia sp. V4 demonstrated a high homology. However, a comparison of Serratia sp. V4 serratiochelin cluster organization with clusters, identified in S. marcescens SM6 and SR 41-8000 strains revealed significant differences.

Conclusion: Both strains S. marcescens SM6 and SR 41-8000 encode NRPS needed for serratiochelin production. The organization of serratiochelin gene clusters in environmental and clinical strains of $S$. marcescens differs from Serrati a sp. V4.

Acknowledgements: This work was supported by the Russian Science Foundation project 16-14-10200 and performed in accordance with the Russian Government Program of Competitive Growth of Kazan Federal University.

\section{References}

1. Crosa J.H., Walsh C.T. (2002) Genetics and assembly line enzymology of siderophore biosynthesis in bacteria. Microbiol. Mol. Biol. Rev. 66(2):223-249.

2. Weber T. et al. (2015) AntiSMASH 3.0 - a comprehensive resource for the genome mining of biosynthetic gene clusters. Nucleic Acids Res. doi 10.1093/nar/gkv437.

3. Seyedsayamdost M.R. et al. (2012) Mixing and matching siderophore clusters: structure and biosynthesis of serratiochelins from Serratia sp. V4. J. Am. Chem. Soc. 134(33):13550-13553. DOI 10.1021/ ja304941d. 